\begin{tabular}{lll} 
Universidade do Minho & Universidade do Minho \\
Escola de Ciências & $\begin{array}{l}\text { Universidade do Minho } \\
\text { Escola de Ciências }\end{array}$ \\
Centro de Matemática & Departamento de Matemática e Aplicações \\
\hline
\end{tabular}

\title{
Mathematica Tools for Quaternionic Polynomials
}

\author{
M. Irene Falcão ${ }^{a, c} \quad$ Fernando Miranda $^{a, c} \quad$ Ricardo Severino $^{c} \quad$ M. Joana Soares ${ }^{b, c}$ \\ a CMAT - Centre of Mathematics, University of Minho, Portugal \\ ${ }^{b}$ NIPE - Economic Policies Research Unit, University of Minho, Portugal \\ ${ }^{c}$ Department of Mathematics and Applications, University of Minho, Portugal
}

\section{Information}

Keywords:

Quaternions, polynomial ring, factorization, symbolic computation.

Original publication:

Lecture Notes in Computer Science, vol. 10405, pp. 394-408, 2017

DOI: $10.1007 / 978-3-319-62395-5 \_27$

www.springerlink.com

\begin{abstract}
In this paper we revisit the ring of (left) one-sided quaternionic polynomials with special focus on its zero structure. This area of research has attracted the attention of several authors and therefore it is natural to develop computational tools for working in this setting. The main contribution of this paper is a Mathematica collection of functions QPolynomial for solving polynomial problems that we frequently find in applications.
\end{abstract}

\section{Introduction}

In this paper we are going to study polynomials in one formal variable $x$ whose coefficients are quaternions located on the left side of the powers of $x$, i.e. polynomials of the form

$$
P(x)=a_{n} x^{n}+a_{n-1} x^{n-1}+\cdots+a_{1} x+a_{0}, \quad a_{k} \in \mathbb{H} .
$$

If $Q(x)=b_{n} x^{n}+b_{n-1} x^{n-1}+\cdots+b_{1} x+b_{0}$ is another polynomial of the same kind, then one can define the operation:

$$
P(x)+Q(x)=\left(a_{n}+b_{n}\right) x^{n}+\left(a_{n-1}+b_{n-1}\right) x^{n-1}+\cdots+\left(a_{1}+b_{1}\right) x+a_{0}+b_{0},
$$

so that the polynomials (1) form an additive abelian group. There are several ways of defining the multiplication such that this group is made a ring. For details on the theory of non-commutative polynomials, we refer the reader to the well-known work of Ore [23]. In the classical case and in many pratical applications (e.g. $[11,25,26])$, one assumes that the variable $x$ commutes with the coefficients, i.e. the multiplication of two polynomials $P$ and $Q$ of degrees $n$ and $m$, respectively, is defined as

$$
P(x) Q(x)=\sum_{k=0}^{m+n}\left(\sum_{j=0}^{k} a_{j} b_{k-j}\right) x^{k},
$$

with the implicit assumption that $a_{k}=0$, if $k>n$ and $b_{k}=0$, if $k>m$. In other words, the set of polynomials of the form (1) endowed with the aforementioned addition and multiplication is a polynomial ring over $\mathbb{H}$ and 
is denoted by $\mathbb{H}[x]$. In the literature, $\mathbb{H}[x]$ is usually referred to as the ring of (left) one-sided, unilateral, basic, simple or standard polynomials and the determination and classification of their zeros have attracted the attention of several authors over the years (see e.g. $[1,2,6,12,13,14,17,18,22,27,29,30,31]$ ) due to the appearance of new interesting algebraic and computational properties.

General polynomials are defined as finite sums of noncommutative monomials of type $a_{0} x a_{1} x \ldots x a_{n}$. General polynomials have been also considered in several works $([7,14,28])$ specially the cases of two-sided or quadratic polynomials (e.g. [16, 20,32]).

In this paper we describe a Mathematica tool for polynomial manipulation in $\mathbb{H}[x]$. The paper is organized as follows. In Sect. 2 we recall well-known concepts and review important results on quaternionic polynomials. In Sect. 3 we focus on the zeros of a polynomial and the relation to its factorization(s) in linear terms. Finally, in Sect. 4 the new Mathematica tools are presented.

\section{The Ring of Quaternionic Polynomials}

In 1843, Hamilton introduced numbers of the form

$$
x=x_{0}+\mathbf{i} x_{1}+\mathbf{j} x_{2}+\mathbf{k} x_{3}, x_{i} \in \mathbb{R},
$$

where $\mathbf{i}, \mathbf{j}$ and $\mathbf{k}$ satisfy the multiplication rules

$$
\mathbf{i}^{2}=\mathbf{j}^{2}=\mathbf{k}^{2}=-1 \text { and } \mathbf{i j}=-\mathbf{j i}=\mathbf{k} .
$$

This non-commutative product generates the algebra of real quaternions $\mathbb{H}$ (named after Hamilton). For a quaternion $x$ of the form (3) we can define, in analogy with the complex case, the real part of $x, \operatorname{Re} x:=x_{0}$, the vector part of $x, \operatorname{Vec} x:=\mathbf{i} x_{1}+\mathbf{j} x_{2}+\mathbf{k} x_{3}$ and the conjugate of $x, \bar{x}:=x_{0}-\mathbf{i} x_{1}-\mathbf{j} x_{2}-\mathbf{k} x_{3}$. The norm of $x$ is given by $|x|:=\sqrt{x \bar{x}}=\sqrt{\bar{x} x}=\sqrt{x_{0}^{2}+x_{1}^{2}+x_{2}^{2}+x_{3}^{2}}$. It immediately follows that each non-zero $x \in \mathbb{H}$ has an inverse given by $x^{-1}=\frac{\bar{x}}{|x|^{2}}$ and therefore $\mathbb{H}$ is a non-commutative division ring or a skew field.

Let us review now some well-known facts on quaternionic polynomials and fix some notation. In what follows, $q$ denotes a quaternion, $P$ is a left one-sided polynomial and $\mathbf{Z}_{P}$ denotes the zero-set of $P$.

Definition 1. (Similarity) Two quaternions $q$ and $q^{\prime}$ are called similar, $q \sim q^{\prime}$, if there exists $h \neq 0$ such that $q^{\prime}=h q h^{-1}$. Similarity is an equivalence relation and the similarity class of $q$, denoted by $[q]$, is the set $[q]=\left\{q^{\prime} \in \mathbb{H}: q \sim q^{\prime}\right\}$.

It can easily be shown (see, e.g. [3]) that

$$
[q]=\left\{q^{\prime} \in \mathbb{H}: \operatorname{Re} q=\operatorname{Re} q^{\prime} \text { and }|q|=\left|q^{\prime}\right|\right\} .
$$

Example 1. The similarity class of $1+\mathbf{i}+\mathbf{j}+\mathbf{k}$ is the three-dimensional sphere in the hyperplane $\left\{(1, x, y, z) \in \mathbb{R}^{4}\right\}$, with center $(1,0,0,0)$ and radius $\sqrt{3}$, i.e.

$$
[1+\mathbf{i}+\mathbf{j}+\mathbf{k}]=\left\{1+\mathbf{i} x+\mathbf{j} y+\mathbf{k} z \in \mathbb{H}: x^{2}+y^{2}+z^{2}=3\right\}
$$

Definition 2. (Special polynomials)

1. The conjugate of $P$ is the polynomial

$$
\bar{P}(x):=\bar{a}_{n} x^{n}+\bar{a}_{n-1} x^{n-1}+\cdots+\bar{a}_{1} x+\bar{a}_{0} .
$$

2. The characteristic polynomial of $q$ is the polynomial

$$
\Psi_{q}(x):=(x-q)(x-\bar{q})=x^{2}-2 \operatorname{Re}(q) x+|q|^{2} .
$$

3. The companion polynomial of $P$ is the real polynomial

$$
\mathcal{C}_{P}(x):=P(x) \bar{P}(x)=\bar{P}(x) P(x) .
$$


Remark 1. The characteristic polynomial of $q$ depends only on the real part and norm of $q$ and, if $q \in \mathbb{H} \backslash \mathbb{R}$, then $\Psi_{q}$ is an irreducible quadratic trinomial from the ring of polynomials $\mathbb{R}[x]$.

Remark 2. In [17], Janovská and Opfer had called the polynomial

$$
\sum_{k=0}^{2 n}\left(\sum_{j=\max \{0, k-n\}}^{\min \{k, n\}} \bar{a}_{j} a_{k-j}\right) x^{k}
$$

the companion polynomial of the quaternionic polynomial $P$, without mentioning that this is nothing else than the usual product of $\bar{P}$ and $P$ in $\mathbb{H}[x]$ (cf. (2)). As pointed out by these authors, the idea of constructing such a polynomial came from the work of Niven [22], where the construction of the polynomial is described as follows: By replacing the quaternions $a_{k}$ by their conjugates $\bar{a}_{k}$ we obtain a polynomial $\bar{P}$. We multiply this on the right by $P(x)$, and allow $x$ to be commutative with the coefficients. Thus we obtain a polynomial with coefficients in $\mathbb{R}$.

In the literature one can find different designations to the polynomial (6), namely basic polynomial, semi-norm or symmetrization of a polynomial. We prefer the designation companion polynomial due to its connection (not recognized explicitly in [17]) to the characteristic polynomial of the companion matrix which was already apparent from the work of [29] (see also [4]).

Example 2. For $P(x)=x^{2}+(2-\mathbf{i}) x+\mathbf{j}+\mathbf{k}$ and $q=\mathbf{i}+\mathbf{j}$ we have

$$
\begin{aligned}
& \Psi_{q}(x)=x^{2}+2 \\
& \mathcal{C}_{P}(x)=\left(x^{2}+(2-\mathbf{i}) x+\mathbf{j}+\mathbf{k}\right)\left(x^{2}+(2+\mathbf{i}) x-\mathbf{j}-\mathbf{k}\right)=x^{4}+4 x^{3}+5 x^{2}+2 .
\end{aligned}
$$

Definition 3. (Evaluation map and zeros)

1. The evaluation of $P$ at $q$ is defined as $P(q):=a_{n} q^{n}+a_{n-1} q^{n-1}+\cdots+a_{1} q+a_{0}$.

2. A quaternion $q$ is a zero (or a root) of $P$, if $P(q)=0$.

3. A zero $q$ is called an isolated zero of $P$, if $[q]$ contains no other zeros of $P$.

4. A zero $q$ is called a spherical zero of $P$, if $q$ is not an isolated zero; $[q]$ is referred to as a sphere of zeros. $^{1}$

Remark 3. We recall here the well-known fact that the evaluation map (at a quaternion $q$ ) is not an algebra homomorphism. In fact, if $P(x)=L(x) R(x)$, then (see e.g. [19])

$$
P(q)=\left\{\begin{array}{l}
0, \quad \text { if } R(q)=0, \\
L(\tilde{q}) R(q), \quad \text { if } R(q) \neq 0,
\end{array} \quad \text { where } \quad \tilde{q}=R(q) q(R(q))^{-1} .\right.
$$

In particular, if $q$ is a zero of $P$ which is not a zero of $R$, then $\tilde{q}$ is a zero of $L$. Next example illustrates this remark.

Example 3. Consider the polynomial $P$ and the quaternion $q$ of last example. If $Q(x)=x-q$, then we have

$$
P(q)=-1+2 \mathbf{i}+3 \mathbf{j} \quad \text { and } \quad Q(q)=0 .
$$

On the other hand, since

$$
(Q P)(x)=x^{3}+(2-2 \mathbf{i}-\mathbf{j}) x^{2}+(-1-2 \mathbf{i}-\mathbf{j}) x+1-\mathbf{i}+\mathbf{j}-\mathbf{k},
$$

we remark that

$$
(Q P)(q)=-2 \mathbf{k} \neq 0=Q(q) P(q) .
$$

\footnotetext{
${ }^{1}$ In such case, it can be proved that all quaternions in $[q]$ are in fact zeros of $P$ and therefore the choice of the term spherical to designate this type of zeros is natural.
} 


\section{Zeros and Factor-Terms in $\mathbb{H}[x]$}

We list now the main results concerning the zero-structure and the factorization of polynomials in $\mathbb{H}[x]$. For the proofs and other details we refer the reader to $[14,19,23]$.

We recall first that it can be proved (see e.g. [15]) that $\mathbb{H}[x]$ is a principal ideal domain and therefore also left and right division algorithms can be defined.

Theorem 1 (Euclidean division). If $A(x)$ and $B(x)$ are polynomials in $\mathbb{H}[x]$ (with $\operatorname{deg} B \leq \operatorname{deg} A$ and $B \neq 0$ ), then there exist unique $Q(x), Q^{\prime}(x), R(x)$ and $R^{\prime}(x)$ such that

$$
A(x)=Q(x) B(x)+R(x)
$$

and

$$
A(x)=B(x) Q^{\prime}(x)+R^{\prime}(x)
$$

with $\operatorname{deg} R<\operatorname{deg} B$ and $\operatorname{deg} R^{\prime}<\operatorname{deg} B$.

If $R(x)=0$ (resp. $R^{\prime}(x)=0$ ) in (8) (resp. (9)), then $B(x)$ is called a right (resp. left) divisor of $A(x)$. The greatest common (right and left) divisor polynomial of two polynomials can be computed using the Euclidean algorithm, by a basic procedure similar to the one used in the complex setting. In this context we also refer to [5] where issues of division within $\mathbb{H}[x]$ are addressed. In what follows, if nothing is specified, right divisors are always assumed.

Concerning the zero structure, the theory of quaternionic polynomials is very different from that of complex polynomials. Nevertheless, in 1941, Niven [22] proved the Fundamental Theorem of Algebra for quaternionic polynomials, establishing that a non-constant polynomial $P$ in $\mathbb{H}[x]$ has a zero in $\mathbb{H}$. As in the classical case, it is also possible to write $P$ as a product of linear factors; however the link between zeros and factors is not straightforward.

Theorem 2 (Factorization into linear terms $[14,19]$ ). Any monic polynomial $P$ of degree $n \in \mathbb{N}$ in $\mathbb{H}[x]$ admits a factorization into linear factors, i.e. there exist $x_{1}, \ldots, x_{n} \in \mathbb{H}$, such that

$$
P(x)=\left(x-x_{n}\right)\left(x-x_{n-1}\right) \cdots\left(x-x_{1}\right) .
$$

Definition 4. (Chain) In a factorization of $P$ of the form (10), the quaternions $x_{1}, \ldots, x_{n}$ will be called factor-terms of $P$ and the $n$-uple $\left(x_{1}, \ldots, x_{n}\right)$ will be called a factor-terms chain associated with $P$ or simply a chain of $P$.

Theorem $3([19,30])$. Let $\left(x_{1}, \ldots, x_{n}\right)$ be a chain of a polynomial $P$. Then every zero of $P$ is similar to some factor-term $x_{k}$ in the chain and reciprocally every factor-term $x_{k}$ is similar to some zero of $P$.

Remark 4. If $\left(y_{1}, \ldots, y_{n}\right)$ is another chain of $P$, then there exists a permutation $\pi$ of $(1, \ldots, n)$ and $h_{1}, \ldots, h_{n} \in \mathbb{H}$, such that $y_{\pi(i)}=h_{i} x_{i} h_{i}^{-1} ; i=1, \ldots n$, i.e. $\left[y_{\pi(i)}\right]=\left[x_{i}\right]$. The explicit expression of $h_{i}$ will be given later on. In such cases we write $\left(x_{1}, \ldots, x_{n}\right) \sim\left(y_{1}, \ldots, y_{n}\right)$ and say that the chains $\left(x_{1}, \ldots, x_{n}\right)$ and $\left(y_{1}, \ldots, y_{n}\right)$ are equivalent or similar.

The explicit relation between factor-terms and zeros of a quaternionic polynomial is addressed in the following results. The first result is useful if one knows a factorization of the polynomial; for example in [10] a numerical method for computing a Weierstrass factorization of a quaternionic polynomial is proposed and the non-spherical zeros are obtained via (11). The second result plays an important role in the construction of polynomials with prescribed zeros.

Theorem 4 (Zeros from factors [19]). Consider a chain $\left(x_{1}, \ldots, x_{n}\right)$ of a polynomial $P$. If the similarity classes $\left[x_{k}\right]$ are distinct, then $P$ has exactly $n$ zeros $\zeta_{k}$ which are related to the factor-terms $x_{k}$ as follows:

$$
\zeta_{k}=\overline{\mathcal{P}}_{k}\left(x_{k}\right) x_{k}\left(\overline{\mathcal{P}}_{k}\left(x_{k}\right)\right)^{-1} ; k=1, \ldots, n,
$$

where

$$
\mathcal{P}_{k}(x):=\left\{\begin{array}{l}
1, \text { if } k=1 \\
\left(x-x_{k-1}\right) \ldots\left(x-x_{1}\right), \text { otherwise }
\end{array}\right.
$$


Theorem 5 (Factors from zeros [1]). If $\zeta_{1}, \ldots, \zeta_{n}$ are quaternions such that the similarity classes $\left[\zeta_{k}\right]$ are distinct, then there is a unique polynomial $P$ of degree $n$ with zeros $\zeta_{1}, \ldots, \zeta_{n}$, which can be constructed from the chain $\left(x_{1}, \ldots, x_{n}\right)$, where

$$
x_{k}=\mathcal{P}_{k}\left(\zeta_{k}\right) \zeta_{k}\left(\mathcal{P}_{k}\left(\zeta_{k}\right)\right)^{-1} ; k=1, \ldots, n
$$

and $\mathcal{P}_{k}$ is the polynomial (12).

In $[1,13]$ more general forms of Theorems 4 and 5 can be found, where the notion of multiplicity of a zero plays a fundamental role. The following result motivates the definition of multiplicity we are going to adopt (in this context we refer also to $[1,2,9,24]$ ).

Theorem $6([1,24])$. Let $P$ be a quaternionic polynomial with degree $n$. Then $x_{1} \in \mathbb{H} \backslash \mathbb{R}$ is the unique zero of $P$ if and only if $P$ admits a unique chain $\left(x_{1}, \ldots, x_{n}\right)$ which has the property ${ }^{2}$

$$
x_{l} \in\left[x_{1}\right] \text { and } x_{l} \neq \bar{x}_{l-1}
$$

for all $l=2, \ldots, n$.

Definition 5. (Multiplicity) The multiplicity of a zero $q$ of $P, m_{P}(q)$, is defined as the maximum degree of the right factors of $P$ having $q$ as their unique zero. The multiplicity of a sphere of zeros $[q]$ of $P, m_{P}([q])$, is the largest $k \in \mathbb{N}_{0}$ for which $\Psi_{q}^{k}$ divides $P$.

Example 4. The polynomial $P(x)=(x-\mathbf{k})(x-\mathbf{j})(x-\mathbf{i})$ has $\mathbf{i}$ as its unique zero of multiplicity 3 . Concerning the polynomial $Q(x)=(x+\mathbf{i})(x-\mathbf{i})(x-\mathbf{i})$ we observe that $m_{Q}(\mathbf{i})=2$ while $m_{Q}([\mathbf{i}])=1$. In [9] such a zero has been called a mixed zero. ${ }^{3}$

The final results of this section are presented/rewritten by taking into account the purpose of the present paper.

Theorem 7. Consider a chain $\left(x_{1}, \ldots, x_{l-1}, x_{l}, \ldots, x_{n}\right)$ of a polynomial $P$.

1. If $h:=\bar{x}_{l}-x_{l-1} \neq 0$ then

$$
\left(x_{1}, \ldots, h^{-1} x_{l} h, h^{-1} x_{l-1} h, \ldots, x_{n}\right) \sim\left(x_{1}, \ldots, x_{l-1}, x_{l}, \ldots, x_{n}\right) .
$$

2. If the chain $\left(x_{1}, \ldots, x_{n}\right)$ has the property (14) and $Q$ is a polynomial of degree $m$ in $\mathbb{H}[x]$ such that $y_{1} \in \mathbb{H} \backslash \mathbb{R}$ is its unique zero and $y_{1} \notin\left[x_{1}\right]$, then the polynomial of degree $n+m, Q P$, has only two zeros, namely $x_{1}$ and $\bar{P}\left(y_{1}\right) y_{1}\left(\bar{P}\left(y_{1}\right)\right)^{-1}$.

Proof. The first result follows by simple manipulation (see also [30] for a different, but equivalent result).

By Theorem 6, the polynomial $Q$ can be written as

$$
Q(x)=\left(x-y_{m}\right) \ldots\left(x-y_{2}\right)\left(x-y_{1}\right), y_{l} \in\left[y_{1}\right], y_{l} \neq \bar{y}_{l-1}, l=2, \ldots, m .
$$

and therefore

$$
T(x):=Q(x) P(x)=\left(x-y_{m}\right) \ldots\left(x-y_{2}\right)\left(x-y_{1}\right)\left(x-x_{n}\right) \ldots\left(x-x_{2}\right)\left(x-x_{1}\right) .
$$

It is clear that $x_{1}$ is a zero of $T$ with multiplicity $n$. Multiplying both sides of last expression by $\bar{P}(x)$ and recalling (5) and (14) we obtain

$$
\begin{aligned}
T(x) \bar{P}(x) & =\left(x-y_{m}\right) \ldots\left(x-y_{2}\right)\left(x-y_{1}\right) \Psi_{x_{1}}^{n}(x) \\
& =\Psi_{x_{1}}^{n}(x)\left(x-y_{m}\right) \ldots\left(x-y_{2}\right)\left(x-y_{1}\right) .
\end{aligned}
$$

Since $\Psi_{x_{1}}(q) \neq 0$ if $q \notin\left[x_{1}\right]$, we conclude that $y_{1}$ is the only zero of $T(x) \bar{P}(x)$ which is not in $\left[x_{1}\right]$. From $\bar{P}\left(y_{1}\right) \neq 0$ and $(7)$, it follows at once that the only zero of $T$ apart from $x_{1}$ is $\bar{P}\left(y_{1}\right) y_{1}\left(\bar{P}\left(y_{1}\right)^{-1}\right)$.

\footnotetext{
${ }^{2}$ In [2], such a chain was called a spherical chain.

${ }^{3}$ More precisely, $q$ is a mixed zero of $P$ if $m_{P}([q])>0$ and $m_{P}(q)>m_{P}\left(q^{\prime}\right)$, for all $q^{\prime} \in[q]$.
} 
The repeated use of Theorem 7 allows to classify (and identify) the zeros of a polynomial from one of its factorization.

Example 5. Consider the polynomial $P$ associated with the chain

$$
(5 \mathbf{j}-5 \mathbf{k}, 5 \mathbf{j},-7 \mathbf{j}+\mathbf{k}, 1+\mathbf{i},-3 \mathbf{i}-4 \mathbf{j}, 3 \mathbf{j}-4 \mathbf{k}) .
$$

The repeated use of (15) allows to state that this chain is equivalent to the following ones:

$$
\begin{gathered}
(5 \mathbf{j}-5 \mathbf{k},-5 \mathbf{j}+5 \mathbf{k}, 3 \mathbf{j}-4 \mathbf{k}, 1+\mathbf{i},-3 \mathbf{i}-4 \mathbf{j}, 3 \mathbf{j}-4 \mathbf{k}) \\
\left(5 \mathbf{j}-5 \mathbf{k},-5 \mathbf{j}+5 \mathbf{k}, 1-\frac{23}{27} \mathbf{i}-\frac{2}{27} \mathbf{j}-\frac{14}{27} \mathbf{k}, \frac{50}{27} \mathbf{i}+\frac{83}{27} \mathbf{j}-\frac{94}{27} \mathbf{k},-3 \mathbf{i}-4 \mathbf{j}, 3 \mathbf{j}-4 \mathbf{k}\right) .
\end{gathered}
$$

Therefore

- $5 \mathbf{j}-5 \mathbf{k}$ is a spherical zero of $P$ with multiplicity 1 ;

- $1-\frac{23}{27} \mathbf{i}-\frac{2}{27} \mathbf{j}-\frac{14}{27} \mathbf{k}$ is an isolated zero of $P$ with multiplicity 1 ;

- $3 \mathbf{j}-4 \mathbf{k}$ is an isolated zero of $P$ with multiplicity 3 .

Finally, one can construct a polynomial with assigned zeros, by the repeated use of the following result.

Theorem 8. A polynomial having $\zeta_{1}$ and $\zeta_{2}$ as its isolated zeros of multiplicity $n$ and $m$, respectively, and a sphere of zeros $\left[\zeta_{s}\right]$ with multiplicity $k$, can be constructed through the chain

$$
(\underbrace{\zeta_{1}, \ldots, \zeta_{1}}_{n}, \underbrace{\tilde{\zeta}_{2}, \ldots, \tilde{\zeta}_{2}}_{m}, \underbrace{\zeta_{s}, \overline{\zeta_{s}}, \ldots, \zeta_{s}, \overline{\zeta_{s}}}_{2 k}),
$$

where $\tilde{\zeta}_{2}=\mathcal{Q}\left(\zeta_{2}\right) \zeta_{2}\left(\mathcal{Q}\left(\zeta_{2}\right)\right)^{-1}$ and $\mathcal{Q}(x)=\left(x-\zeta_{1}\right)^{n}$.

Proof. The use of Theorem 2.8 in [13], adapted to the case of left one-sided polynomials, allows the construction of a family of polynomials with the assigned zeros, namely $P=\Psi_{\zeta_{s}}^{k}(x)\left(x-y_{m}\right) \ldots\left(x-y_{1}\right)(x-$ $\left.x_{n}\right) \ldots\left(x-x_{1}\right)$, where

- $x_{1}=\zeta_{1}$ and $x_{l}$ are chosen in $\left[x_{1}\right]$, so that $x_{l} \neq \bar{x}_{l-1}, l=2, \ldots, n$. In particular, one can take $x_{n}=\cdots=x_{1}=\zeta_{1}$ in line with the classical definition of multiplicity;

- $\tilde{\zeta}_{2}=y_{1}=\mathcal{P}_{n+1}\left(\zeta_{2}\right) \zeta_{2}\left(\mathcal{P}_{n+1}\left(\zeta_{2}\right)\right)^{-1}$, where $\mathcal{P}_{n+1}$ is given by (12) (cf. (13));

- $y_{k}, k=2, \ldots, m$ are chosen in $\left[y_{1}\right]$, so that $y_{k} \neq \bar{y}_{k-1}$. The particular choice $y_{m}=y_{m-1}=\cdots=$ $y_{1}=\tilde{\zeta}_{2}$ leads to the final result.

Example 6. An example of a polynomial $P$ which has $x_{1}=\mathbf{i}$ as a zero of multiplicity $3, x_{2}=-1+\mathbf{j}+\mathbf{k}$ as a zero of multiplicity 2 and $[2+\mathbf{i}]$ as a sphere of zeros with multiplicity 2 is:

$$
P(x)=\Psi_{2+\mathbf{i}}^{2}\left(x-\tilde{x}_{2}\right)^{2}\left(x-x_{1}\right)^{3},
$$

where $\tilde{x}_{2}=\mathcal{P}_{4}\left(x_{2}\right) x_{2}\left(\mathcal{P}_{4}\left(x_{2}\right)\right)^{-1}$ and $\mathcal{P}_{4}(x)=\left(x-x_{1}\right)^{3}$, i.e.

$$
P(x)=(x-2-\mathbf{i})^{2}(x-2+\mathbf{i})^{2}\left(x+1+\frac{7}{5} \mathbf{i}+\frac{1}{5} \mathbf{j}\right)^{2}(x-\mathbf{i})^{3} .
$$

It follows immediately from the above considerations that the polynomial

$$
Q(x)=(x-2-\mathbf{i})^{2}(x-2+\mathbf{i})^{2}(x+1-\mathbf{j}-\mathbf{k})\left(x+1+\frac{7}{5} \mathbf{i}+\frac{1}{5} \mathbf{j}\right)(x-\mathbf{k})(x-\mathbf{j})(x-\mathbf{i})
$$

solves the same problem.

\footnotetext{
${ }^{4}$ In [2] the same problem is also addressed and one can find a particular (different) choice of $\tilde{x}_{l}(l=2, \ldots, n)$ and $y_{l}$ $(l=2, \ldots, m)$.
} 


\section{QPolynomial}

Some years ago, two of the authors of this paper introduced some new tools on the Mathematica standard package Quaternions for implementing Hamilton's Quaternion Algebra [8]. Later on, a new add-on QuaternionAnalysis [21] was developed to provide also tools for manipulating regular quaternion valued functions. This package can be downloaded at the Wolfram Library archive and full description of the QuaternionAnalysis functions as well as illustrative examples can be found in the tutorial included in [21].

QPolynomial is a collection of Mathematica functions, depending on the package QuaternionAnalysis, for treating usual problems in $\mathbb{H}[x]$ : evaluation, euclidean division, greatest common divisor polynomial, construction of a polynomial with prescribed zeros, etc. In this context, a quaternionic polynomial is an object of the form Polynomial $\left[a_{n}, a_{n-1}, \ldots, a_{1}, a_{0}\right]$ accordingly to (1). For such objects, rules as Plus, NonCommutativeMultiply, Power and functions as Conjugate, Eval, CharacteristicPolynomial, CompanionPolynomial, etc., are defined. We summarize in Table 1 the most important functions included in QPolynomial. Auxiliary functions which come with its own usefulness are also listed in the same table. The use of these functions is illustrated by several examples.

Example 7. Rules on the object Polynomial

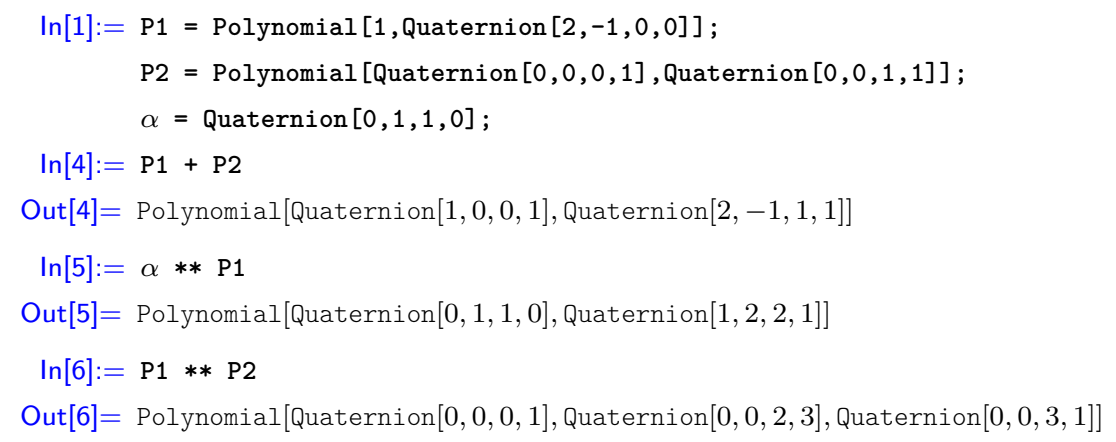

Example 8. Polynomial Functions (cf. Example 2)

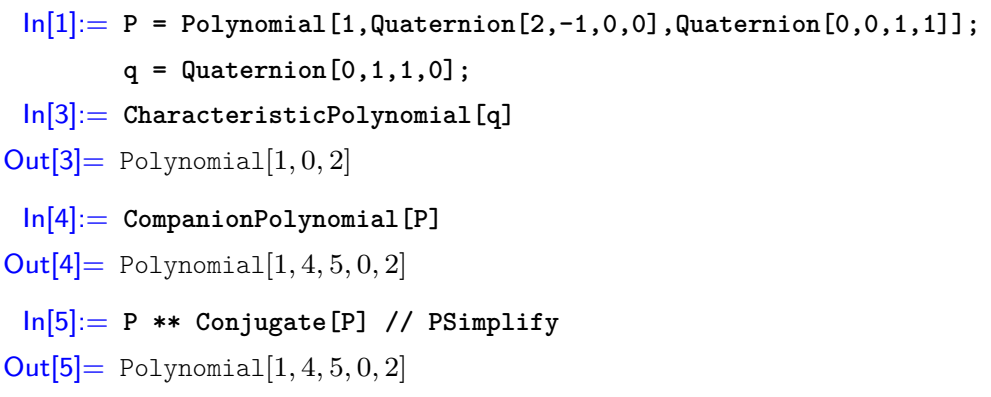

Example 9. Evaluation and zeros (cf. Example 3)

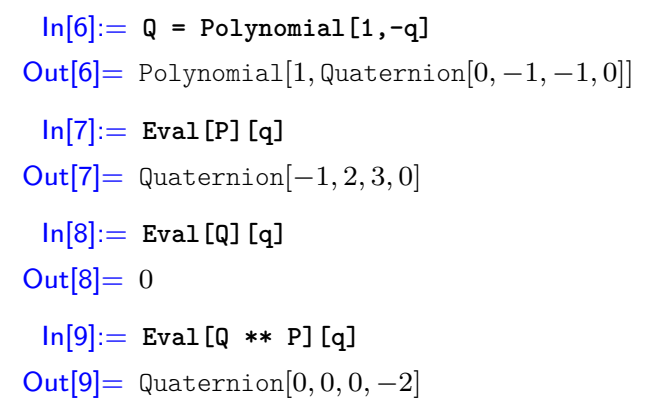

Next example addresses issues of division in $\mathbb{H}[x]$. Both left and right Euclidean division are implemented in QPolynomial (see Theorem 1). 

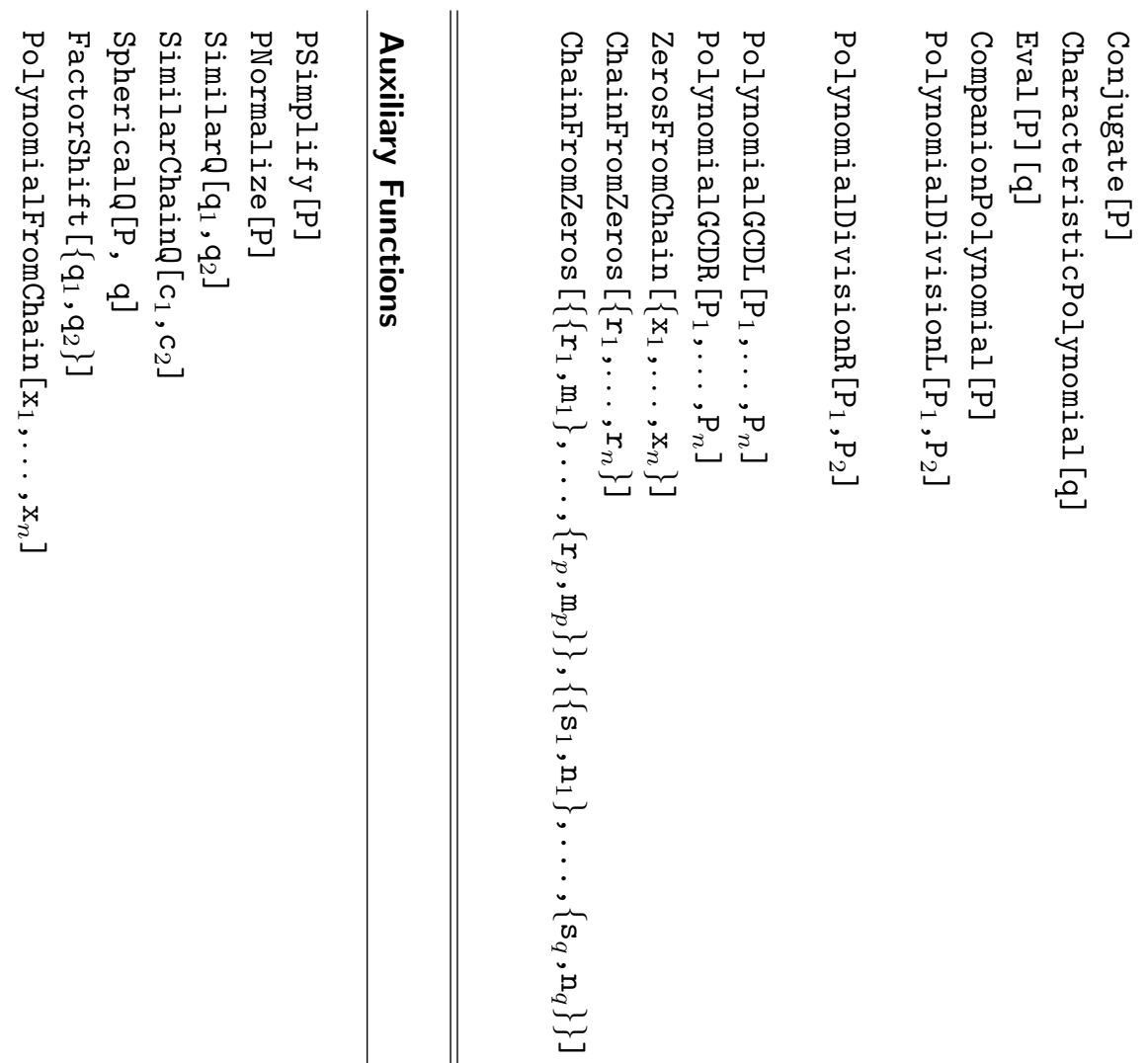

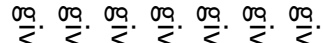

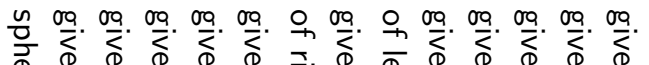
更. 商家

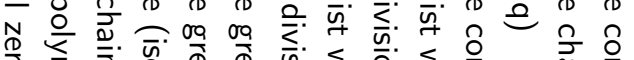

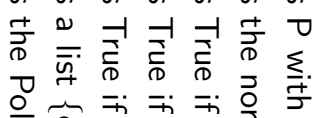

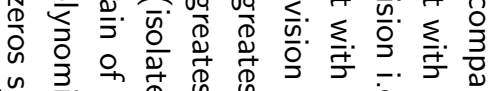

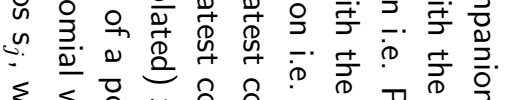

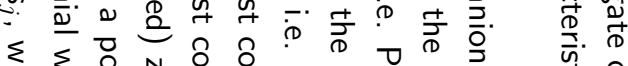
主势号

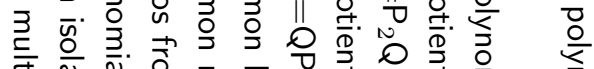

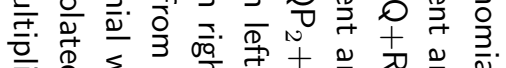

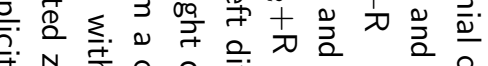

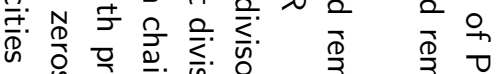

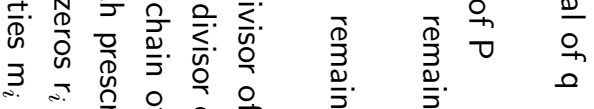

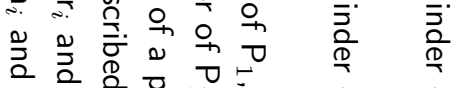

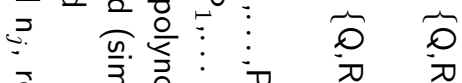

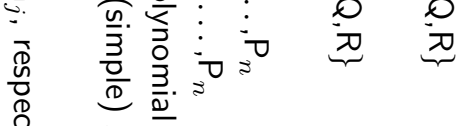


Example 10. Euclidean division and GCD

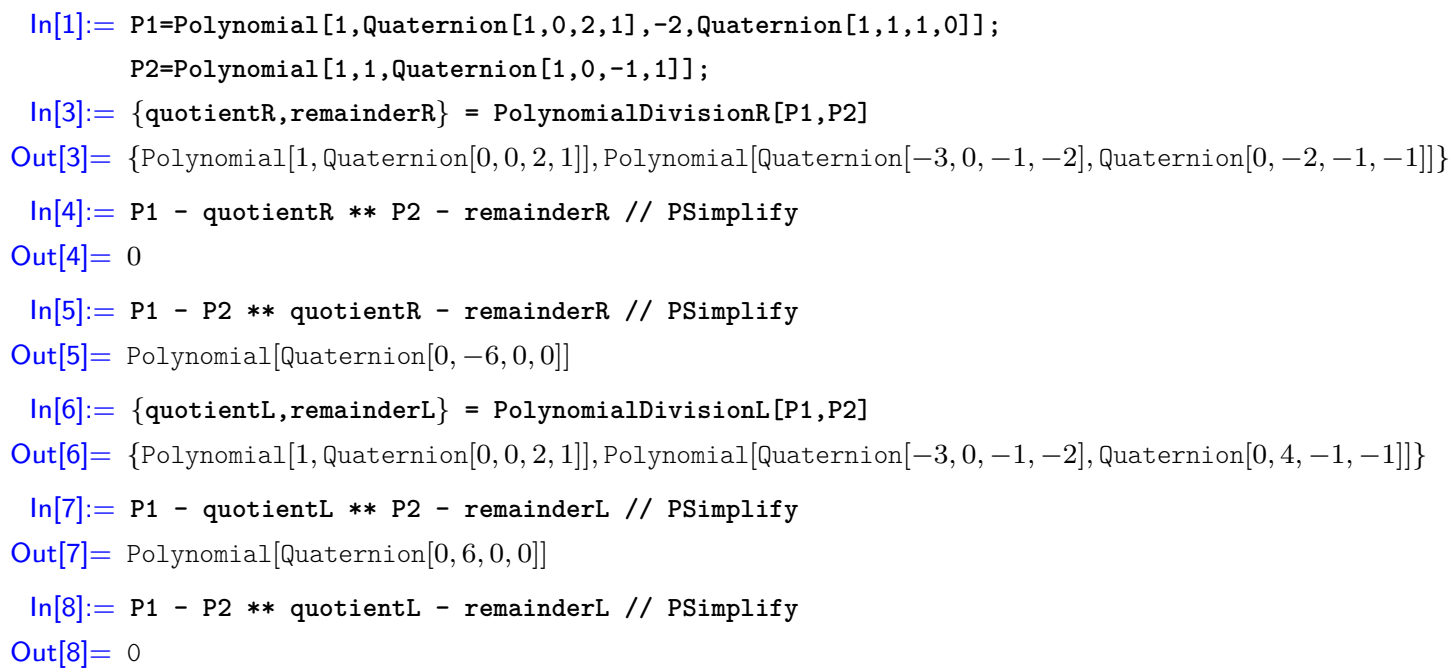

In the quaternion case, greatest common left and right divisors are computed by a procedure similar to the classical Euclidean algorithm via PolynomialGCDL and PolynomialGCDR, respectively.

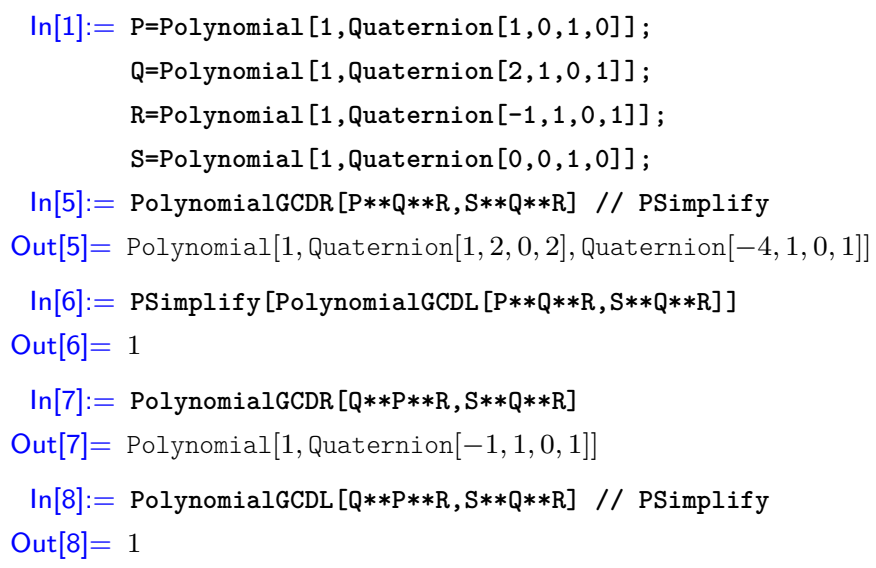

For complex arguments, PolynomialDivisionL and PolynomialDivisionR coincide with the PolynomialQuotientRemainder built-in function and the same is true for PolynomialGCDL, PolynomialGCDR and PolynomialGCD.

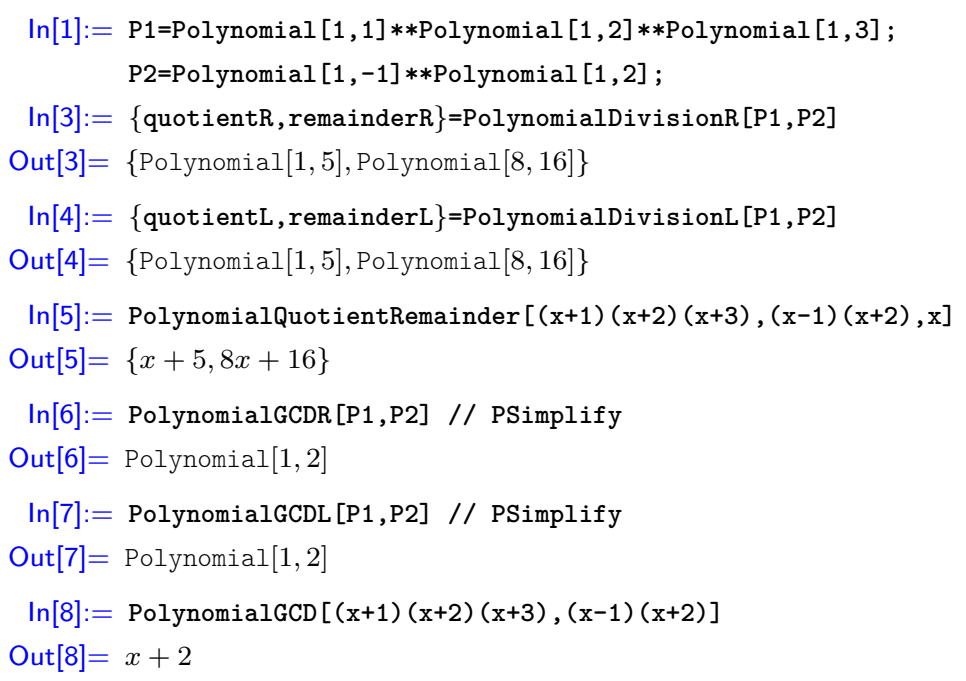

The functions ZerosFromChain and ChainFromZeros are based on the use of Theorem 4 and Theorem 5, respectively. 
Example 11. Factors from zeros and zeros from factors

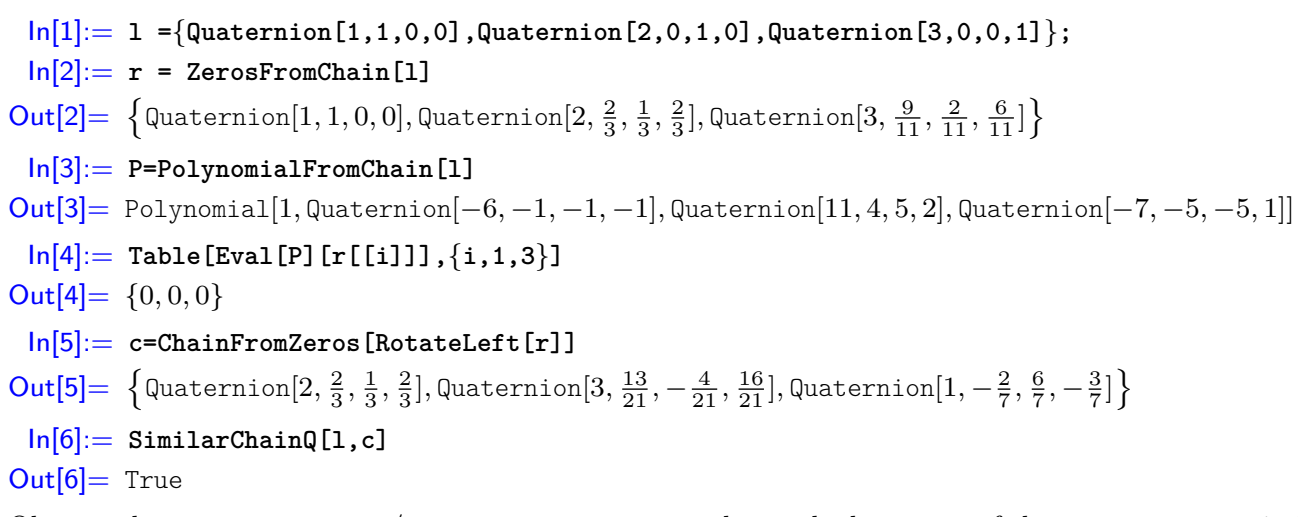

Observe that ZerosFromChain/ChainFromZeros can not be used when some of the arguments are similar. This problem can be overcome in the case of ChainFromZeros by using an alternative argument syntax (see Example 13).

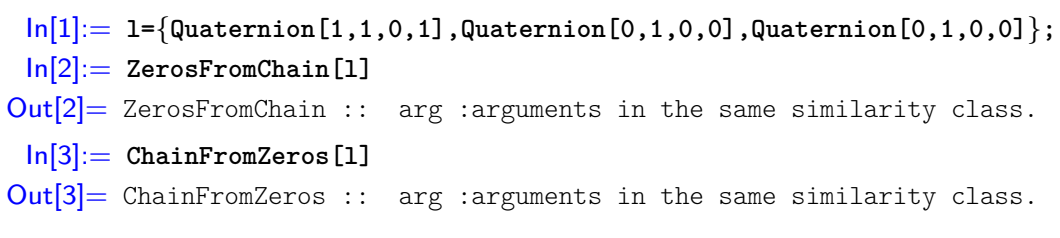

Use an alternative syntax.

Next example shows how to use the function FactorShift for successive applications of (15).

Example 12. Identifying the zeros from a given chain (cf. Example 5)

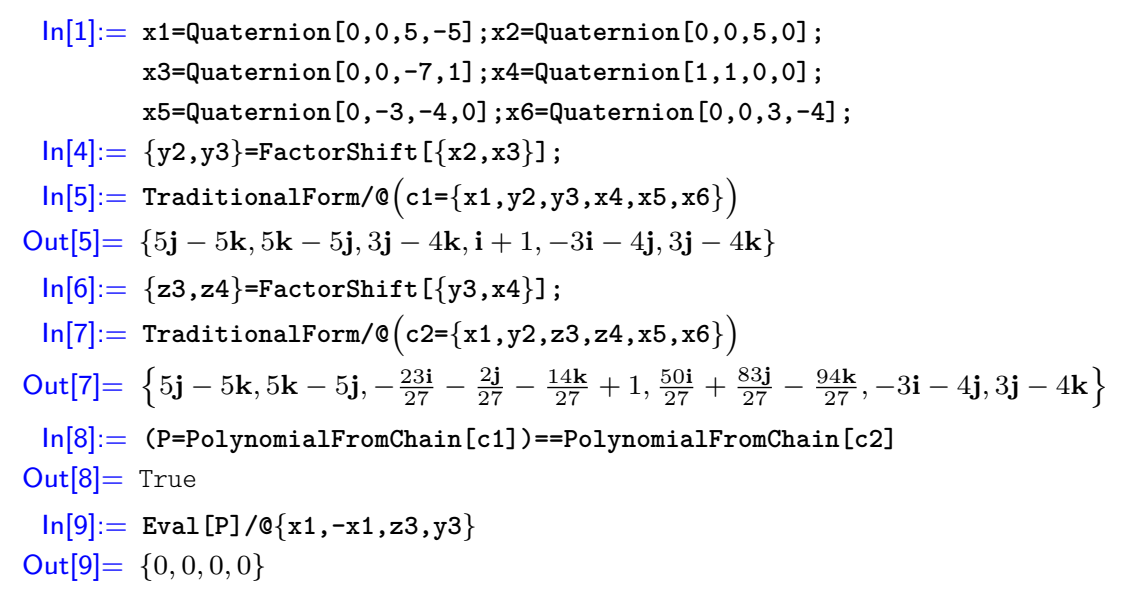

Our last example illustrates the use of the function ChainFromZeros which allows the construction of a polynomial with assigned zeros and corresponding multiplicities. It is based on the repeated use of Theorem 8.

Example 13. Polynomial with prescribed zeros (cf. Example 6)

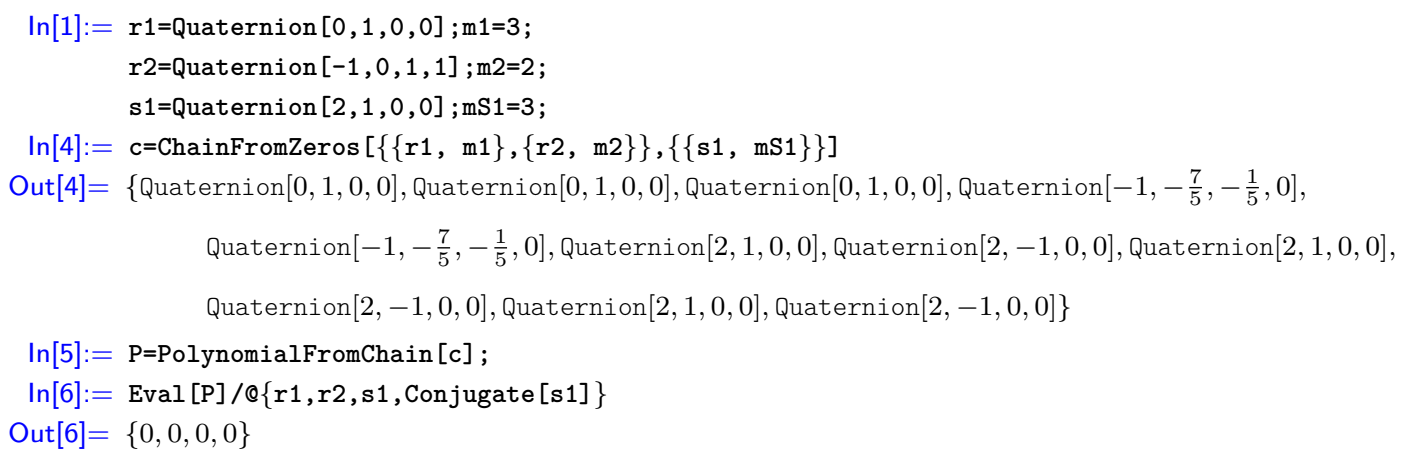




\subsubsection{Acknowledgments.}

Research at CMAT was financed by Portuguese funds through Fundação para a Ciência e a Tecnologia, within the Project UID/MAT/00013/2013. Research at NIPE was carried out within the funding with COMPETE reference number POCI-01-0145FEDER-006683 (UID/ECO/ 03182/2013), with the FCT/MEC's (Fundação para a Ciência e a Tecnologia, I.P.) financial support through national funding and by the ERDF through the Operational Programme on "Competitiveness and Internationalization COMPETE 2020" under the PT2020 Partnership Agreement.

\section{References}

[1] Beck, B.: Sur les équations polynomiales dans les quaternions. Enseign. Math. 25, 193-201 (1979)

[2] Bolotnikov, V.: Zeros and factorizations of quaternion polynomials: the algorithmic approach. arXiv:1505.03573 (2015)

[3] Brenner, J.L.: Matrices of quaternions. Pacific J. Math. 1(3), 329-335 (1951)

[4] Chapman, A., Machen, C.: Standard polynomial equations over division algebras. Adv. Appl. Clifford Algebras (2016), doi:10.1007/s00006-016-0740-4

[5] Damiano, A., Gentili, G., Struppa, D.: Computations in the ring of quaternionic polynomials. J. Symbolic Comput. 45(1), 38-45 (2010)

[6] De Leo, S., Ducati, G., Leonardi, V.: Zeros of unilateral quaternionic polynomials. Electron. J. Linear Algebra 15, 297-313 (2006)

[7] Eilenberg, S., Niven, I.: The "fundamental theorem of algebra" for quaternions. Bull. Amer. Math. Soc. 50, $246-248$ (1944)

[8] Falcão, M.I., Miranda, F.: Quaternions: A Mathematica package for quaternionic analysis. Lecture Notes in Comput. Sci. 6784, 200-214 (2011)

[9] Falcão, M.I., Miranda, F., Severino, R., Soares, M.J.: Quaternionic polynomials with multiple zeros: A numerical point of view. AIP Conference Proceedings 1798(1), 020099 (2017)

[10] Falcão, M.I., Miranda, F., Severino, R., Soares, M.J.: Weierstrass method for quaternionic polynomial root-finding. arXiv:1702.04935 (2017)

[11] Farouki, R.T., Gentili, G., Giannelli, C., Sestini, A., Stoppato, C.: A comprehensive characterization of the set of polynomial curves with rational rotation-minimizing frames. Adv. Comput. Math. 43(1), 1-24 (2017)

[12] Gentili, G., Stoppato, C.: Zeros of regular functions and polynomials of a quaternionic variable. Mich. Math. J. 56(3), 655-667 (2008)

[13] Gentili, G., Struppa, D.C.: On the multiplicity of zeroes of polynomials with quaternionic coefficients. Milan J. Math. 76, 15-25 (2008)

[14] Gordon, B., Motzkin, T.: On the zeros of polynomials over division rings I. Trans. Amer. Math. Soc. 116, $218-226$ (1965)

[15] Jacobson, N.: The Theory of Rings. Mathematical surveys and monographs, American Mathematical Society, New York (1943)

[16] Janovská, D., Opfer, G.: The classification and the computation of the zeros of quaternionic, two-sided polynomials. Numer. Math. 115(1), 81-100 (2010)

[17] Janovská, D., Opfer, G.: A note on the computation of all zeros of simple quaternionic polynomials. SIAM J. Numer. Anal. 48(1), 244-256 (2010)

[18] Kalantari, B.: Algorithms for quaternion polynomial root-finding. J. Complexity 29(3-4), 302-322 (2013)

[19] Lam, T.Y.: A first course in noncommutative rings. Graduate Texts in Mathematics, Springer-Verlag, New York (1991)

[20] Lianggui, F., Kaiming, Z.: Classifying zeros of two-sided quaternionic polynomials and computing zeros of two-sided polynomials with complex coefficients. Pacific J. Math 262(2), 317-337 (2013)

[21] Miranda, F., Falcão, M.I.: QuaternionAnalysis Package: User's Guide, Technical Report (2014) http://w3.math.uminho.pt/QuaternionAnalysis

[22] Niven, I.: Equations in quaternions. Amer. Math. Monthly 48, 654-661 (1941)

[23] Ore, O.: Theory of non-commutative polynomials. Ann. Math. 34(3), 480-508 (1933)

[24] Pereira, R.: Quaternionic Polynomials and Behavioral Systems. Ph.D. Thesis, Universidade de Aveiro (2006)

[25] Pereira, R., Rocha, P.: On the determinant of quaternionic polynomial matrices and its application to system stability. Math Methods Appl Sci. 31(1), 99-122 (2008)

[26] Pereira, R., Rocha, P., Vettori, P.: Algebraic tools for the study of quaternionic behavioral systems. Linear Algebra Appl. 400(1-3), 121-140 (2005)

[27] Pogorui, A., Shapiro, M.: On the structure of the set of zeros of quaternionic polynomials. Complex Var. Theory Appl. 49(6), 379-389 (2004)

[28] Pumplün, S., Walcher, S.: On the zeros of polynomials over quaternions. Commun. Algebra 30(8), 4007-4018 (2002)

[29] Serôdio, R., Pereira, E., Vitória, J.: Computing the zeros of quaternion polynomials. Comput. Math. Appl. 42(8-9), 1229$1237(2001)$

[30] Serôdio, R., Siu, L.S.: Zeros of quaternion polynomials. Appl. Math. Lett. 14(2), 237-239 (2001)

[31] Topuridze, N.: On the roots of polynomials over division algebras. Georgian Math. J. 10(4), 745-762 (2003)

[32] Xu, W., Feng, L., Yao, B.: Zeros of two-sided quadratic quaternion polynomials. Adv. Appl. Clifford Algebras 24(3), 883-902 (2014) 